\title{
Los estudios de Fanny Medina, primera médica colegiada de Andalucía, en la Facultad de Medicina de Cádiz
}

\author{
Víctor M. Heredia Flores \\ Departamento de Teoría e Historia Económica \\ Universidad de Málaga \\ e-mail: vmheredia@uma.es \\ ORCID: https://orcid.org/0000-0002-1030-5735
}

\begin{abstract}
RESUMEN: El conocimiento de los nombres de las mujeres que fueron pioneras en el acceso a estudios superiores y el ejercicio de profesiones liberales entre finales del siglo XIX y principios del XX todavía necesita ser completado. En este artículo se aborda el caso de Francisca Medina Verdeja, una de las primeras mujeres andaluzas en alcanzar la licenciatura y el doctorado en Medicina, a partir de la documentación existente en el Archivo de la Universidad de Cádiz. Asimismo, se confirma que ejerció profesionalmente como médica desde 1921, lo que la convierte en la primera mujer en hacerlo en Andalucía.
\end{abstract}

Palabras clave: Mujeres, Universidad, Medicina, Francisca Medina.

\section{The studies of Fanny Medina, first woman doctor collegiate of Andalusia, at the School of Medicine of Cadiz}

ABSTRACT: Knowledge of the names of the women who pioneered access to higher education and the exercise of the liberal professions between the late 19th and early 20th centuries still needs to be completed. This article deals with the case of Francisca Medina Verdeja, one of the first Andalusian women to complete a degree and a doctorate in Medicine, based on the existing documentation in the Archive of the University of Cadiz. Likewise, it is confirmed that she practiced professionally as a doctor since 1921, which makes her the first woman to do so in Andalusia.

Keywords: Women, University, Medicine, Francisca Medina.

En la situación en la que nos encontramos, ante una inédita — para las generaciones vivas - crisis de carácter sanitario, parece más oportuno aún recordar a personas que han trabajado en este campo de la actividad humana y más aún si tratamos de rescatar la trayectoria de mujeres olvidadas en un oscuro rincón de la memoria colectiva, pero con una existencia cierta. Un nombre femenino que ha permanecido en el olvido, y que por su condición de 
pionera en el ejercicio profesional de la Medicina en Andalucía merece ser recuperado es el de la malagueña Francisca Medina Verdeja, Fanny Medina, que cursó sus estudios universitarios en la Facultad de Cádiz. Situemos primero su caso en el contexto de la incorporación de las mujeres a las carreras superiores en la España de principios del siglo XX.

\section{LA INCORPORACIÓN DE LAS MUJERES A LA UNIVERSIDAD A PRINCIPIOS DEL SIGLO XX}

Actualmente en España ejercen la Medicina más mujeres que hombres. Según datos del Instituto Nacional de Estadística, en el año 2019 había 138.250 médicas (término preferido por la Real Academia Española) sobre un total de 267.995 profesionales colegiados. Es decir, las mujeres representan el 51,6 \%. En 1954 el número de mujeres ejercientes era de solo 334, apenas un uno por ciento de los médicos españoles de aquel momento ${ }^{1}$.

Las historiadoras feministas sobre las Ciencias y la Medicina en España han incidido en los sesgos de género del conocimiento y se han ocupado de recuperar nombres de mujeres pioneras para la historia de las Ciencias en nuestro país. Pero se ha hecho más hincapié en las instituciones que promovieron el conocimiento científico en un medio social y político caracterizado por cierto desinterés, relegando la recuperación de los nombres propios (Santesmases, Cabré y Ortiz 2017). Un ejemplo podría ser el de Trinidad Arroyo, la primera oftalmóloga española, estudiada por Carmen Magallón (2011).

La feminización de la profesión médica ha recorrido un largo camino en el que podemos ubicar los casos de algunas pioneras, como la malagueña Fanny Medina. La capacidad de las mujeres para practicar la Medicina fue muy cuestionada desde el siglo XVIII, a pesar de que existía una tradicional dedicación a la sanación y a las artes curativas de muchas féminas. El pudor, la presunta menor capacidad intelectual y la derivación obligatoria a las tareas del hogar complicaban enormemente la vocación médica de muchas jóvenes.

Por otra parte, el acceso de las mujeres a los estudios universitarios no se hizo significativo hasta la década de 1920, una vez superados los obstáculos legales que dificultaban la incorporación femenina a las facultades. En 1882, cuando ya se había producido la matriculación de las primeras alumnas universitarias, un real decreto dejó en suspenso la admisión de mujeres en la enseñanza superior. Otro real decreto de 1888 abrió de nuevo las puertas de las universidades a las mujeres, pero con la condición de solicitar una autorización expresa para ello, lo que dilataba y complicaba la posibilidad de matricularse en los establecimientos docentes oficiales, ya que la admisión quedaba a la consideración de la autoridad «según el caso y las circunstancias de la interesada».

En todo caso, el número de mujeres que finalizaron estudios universitarios hasta 1910 es muy reducido, apenas algo más de medio centenar (Flecha 1996). En ese año fue definitivamente derogada la necesidad de admisión, dejando el acceso a los estudios en igualdad de condiciones sin distinción de sexos (Rodríguez López 2010). Esto permitió que, en un clima social ligeramente más favorable a la figura de la mujer que cursaba estudios,

\footnotetext{
${ }^{1}$ El INE elabora la Estadística de profesionales sanitarios colegiados desde el año 1952. Los resultados pueden consultarse en: https://www.ine.es/dyngs/INEbase/es/operacion.htm?c=Estadistica

DOI del artículo:

https://doi.org/10.25267/Cuad investig fondos arch UCA.2021.i3.02 
fueran creciendo las alumnas universitarias. En el curso 1914-1915 había 90 mujeres matriculadas en universidades españolas, apenas un $0,45 \%$ del total del alumnado. A partir de ese momento, la incorporación femenina a la enseñanza superior fue progresiva, y en el curso 1932-1933 ya había en España más de 2.000 jóvenes universitarias, un 6,7\% del total (Vázquez Ramil 2012).

Las carreras preferidas por las españolas que podían permitirse estudiar en las primeras décadas del siglo eran Medicina, Farmacia, Ciencias y Filosofía y Letras. Otros estudios no universitarios en los que la presencia de la mujer fue amplia y a veces exclusiva, eran los de matronas, enfermeras, maestras y auxiliares comerciales.

La carrera de Medicina fue la elegida mayoritariamente por las primeras universitarias en España y en otros países. Aun así, hasta 1918, según los datos que Elena Soriano facilitó a la norteamericana Kate Campbell Hurd-Mead, solo doce mujeres finalizaron la licenciatura en Medicina (Cabré 2001, p. 118). A partir de 1910 fue superada en las preferencias femeninas por otras carreras como Ciencias, Farmacia y Filosofía y Letras (Guil y Flecha 2015). Las médicas que ejercían por entonces se especializaban en Ginecología y Pediatría, para las que se les atribuía una sensibilidad adecuada y que les permitían ser aceptadas socialmente a la hora de trabajar. La cirugía, por ejemplo, no se consideraba conveniente para las mujeres (Vázquez Ramil 2012, p. 119).

La carrera de Farmacia fue una de las más feminizadas en aquellas décadas. Se consideraba especialmente apropiada para las mujeres, ya que era «como una forma superior de cocina en la que lo único que había que hacer era seguir al pie de la letra las instrucciones de la receta, y requería de paciencia, de inmovilidad y sosegada reflexión» (Vázquez Ramil 2012, p. 117). Encarnación Lemus (2017) apunta en esta misma dirección al indicar que en la Residencia de Señoritas fueron mayoritarias las farmacéuticas,

algo que nuevamente confirma que Farmacia fue una opción preferente para las mujeres o, tal vez, para los padres de las estudiantes: una licenciatura que las obligaba a volver a sus pueblos para establecerse y que estaba bien vista, ya que su ejercicio no tenía por qué alejarlas de casa.

La cuestión de la validez profesional de los títulos académicos conquistados por mujeres generó intensos debates a finales del siglo XIX. Podían estudiar, pero no ejercer. El dilema se fue resolviendo hacia el reconocimiento en igualdad con los varones, pero de forma lenta. En 1900 se reconoció a María Dolores Martínez Rodríguez, licenciada en Farmacia, que su título la facultaba legalmente para ejercer la profesión de farmacéutica (Flecha 1998). En 1910 una orden disponía que la posesión de títulos académicos facultaba a las mujeres para el ejercicio de cuantas profesiones tuvieran relación con el Ministerio de Instrucción Pública (Rodríguez López 2010).

\section{LAS PRIMERAS MÉDICAS ANDALUZAS}

A la altura de 1909, más de la mitad de las universitarias españolas estaban matriculadas en facultades de Medicina. La primera mujer que se matriculó oficialmente en esta carrera lo había hecho en Barcelona en 1872. Elena Maseras, junto con Dolores Aleu y Martina Castells, 
fueron las primeras en terminar la licenciatura, las tres en la Universidad de Barcelona, aunque solo Dolores Aleu llegó a ejercerla profesionalmente, con consulta abierta en Barcelona durante veinticinco años.

La primera andaluza que estudió Medicina lo hizo en la Facultad de Cádiz. Fue Antonia Monreal y Andrés, quien empezó en 1889 y acabó la licenciatura en 1896 (Flecha 1996, p. 230), aunque no consta que ejerciera profesionalmente. Habrá que esperar a 1911 para que se matricule la primera mujer en la Facultad de Medicina de la Universidad de Granada, Eudoxia Píriz Diego, que concluyó la carrera en 1920. Píriz fue «obligada a repetir el tercer curso de los estudios de licenciatura tras haber sido discriminada por razón de género en un examen final oral ante el catedrático de Farmacología (1916)» (Barranco, en línea). Discípula del catedrático Alejandro Otero, Píriz ha sido considerada como la primera médica del siglo XX en Andalucía, pionera en la región a la hora de ejercer legalmente la profesión, ya que se dio de alta en el Colegio de Médicos granadino en 1923, especializándose en Ginecología y Obstetricia (García Barranco y Barranco Castillo 2002). Este hecho se ha sostenido con rotundidad, incluso afirmando que fue la primera mujer en estudiar Medicina en Andalucía, mérito que cabe atribuir en justicia a Antonia Monreal, quien terminó los estudios casi un cuarto de siglo antes. Y en cuanto a la primera mujer que ejerció de forma profesional y pública la Medicina en la región, parece que esta circunstancia recae en la malagueña Francisca Medina, cuya actividad ya está documentada en 1921.

El criterio de la colegiación para conocer los nombres de las pioneras presenta sus limitaciones, ya que su obligatoriedad se estableció en 1898, pero seis años después se declaró potestativa. El real decreto de 28 de mayo de 1917 sobre los Colegios de Médicos fijó definitivamente la colegiación obligatoria como requisito imprescindible para el ejercicio de la profesión médica. Teresa Ortiz Gómez (1985-86, p. 362), a partir de la documentación de los diferentes colegios de la comunidad, estableció que las primeras colegiadas andaluzas se inscribieron en Granada (1923, la citada Píriz), Sevilla (1925, Carmen Peralta Villar), Málaga (1928, Francisca Medina) y Huelva (1929, Loreto Tapia). La citada autora afirmaba, a partir de la información de la memoria de licenciatura de Esther Rosado Camacho, que «entre 1898 y 1922 no se inscribió ninguna mujer en los Colegios de Médicos de Andalucía, como tampoco acabó sus estudios ninguna mujer en las Facultades de Medicina andaluzas» (Ortiz 1985-1986, p. 347). Pero, como hemos adelantado, esta información venía viciada por la desaparición del expediente de Francisca Medina Verdeja del archivo del Colegio de Médicos de Málaga.

\section{LA ETAPA INICIAL DE FORMACIÓN DE FANNY MEDINA}

Hasta hace muy poco tiempo apenas disponíamos de información acerca de la trayectoria vital de Fanny Medina. Las primeras referencias en el mundo académico proceden de los trabajos de Concepción Campos Luque (2001, p. 174). Esta historiadora localizó en sus investigaciones sobre el mundo del trabajo femenino en la Málaga de la etapa final de la Restauración a esta mujer y publicó las primeras noticias sobre su dedicación profesional a la Medicina como situación excepcional en las primeras décadas del siglo XX. Afortunadamente,

DOI del artículo:

https://doi.org/10.25267/Cuad investig fondos arch UCA.2021.i3.02 
en los últimos meses hemos podido reunir una serie de documentos y noticias que nos permiten ampliar nuestro conocimiento de la biografía y de la dedicación profesional de esta pionera.

El 24 de agosto de 1891, a las seis de la tarde, nacía en la malagueña calle Comedias n. ${ }^{\circ}$ 29 la niña Francisca Guillerma Victoria de los Ángeles Medina Verdeja, como consta en la correspondiente inscripción del Registro Civil. Fue la única hija del matrimonio formado por Francisco Medina Chavarría, natural de Lucena y dedicado al comercio, y la malagueña Victoria Verdeja Maldonado ${ }^{2}$.

Desconocemos dónde realizó los estudios primarios Francisca, que desde pequeña era conocida como Fanny y así firmaba los documentos oficiales. Es muy posible que fuera alumna de alguno de los colegios de monjas, que a la altura de 1900 existían en la ciudad (La Asunción, Sagrada Familia, Esclavas), o que estudiara en algún centro femenino no religioso como el que a finales del siglo XIX regentó Fanny Pezzi de Luque con el nombre de San Rafael en las proximidades del domicilio de los Medina Verdeja. Este colegio aplicaba un sistema pedagógico moderno con el objetivo de «hacer de nuestras discípulas, señoritas modelos de cultura y distinción, a la vez que mujeres honradas, religiosas, fuertes y útiles a la sociedad y a la familia» ${ }^{3}$.

Las primeras referencias ciertas sobre sus estudios corresponden a su matriculación en el Real Conservatorio de Música María Cristina que sostenía la Sociedad Filarmónica de Málaga. Entre los años académicos 1900-1901 y 1905-1906 fue superando, como alumna de enseñanza libre, las asignaturas de cinco cursos de Solfeo y ocho de Piano, siempre con la calificación de sobresaliente ${ }^{4}$. En las actas de los exámenes figura como profesor de Fanny el músico malagueño Juan Cabas Galván, autor de zarzuelas y piezas cómicas, algunas compuestas en unión de su hermano José (López Marinas 2015-2016, pp. 237-238).

Una vez finalizada la carrera de Piano y poco después de cumplir los quince años, el 24 de septiembre de 1906, realizó el ejercicio de ingreso en la Escuela Normal Superior de Maestras. En la solicitud que presentó expresaba que contaba con el consentimiento de su padre, quien así lo hacía constar en el mismo documento. Una vez obtenido el aprobado en el ingreso hizo un nuevo ejercicio para conseguir la nota de sobresaliente con matrícula de honor, que le permitió matricularse gratuitamente en las asignaturas del primer curso, que superó en septiembre de $1907^{5}$.

Entonces continuó su formación en el Instituto General y Técnico de Málaga. Francisca Medina superó los cinco cursos del Bachillerato en tan solo dos años, entre 1907 y 1909, presentándose a los exámenes como alumna libre. En la convocatoria de septiembre de 1907 se examinó de ocho asignaturas y en junio de 1908 se presentó a las pruebas de diez materias, completando las once que le faltaban en 1909. Aprobó los dos ejercicios del grado el 30 de septiembre de 1909, siéndole expedido el título de bachiller en Granada el 8 de agosto de 1911.

\footnotetext{
${ }^{2}$ Registro Civil de Málaga (RCM), sección $1^{\text {a }}$, tomo 63, fol. 192

${ }^{3}$ Se conserva un folleto de este establecimiento publicado en 1900. Archivo Díaz de Escovar-Fundación Unicaja (ADE), caja 36, carpeta 17.

${ }^{4}$ Archivo Histórico Provincial de Málaga (AHPM), Conservatorio. Relaciones de estudios. Tomo III, libro 24.058, p. 271; y Actas de exámenes, años de 1902 a 1907, libro 24.001.

${ }^{5}$ El expediente académico de la Escuela Normal está en el Archivo General de la Universidad de Málaga (AGUMA), signatura S1-M1-110.

DOI del artículo:

https://doi.org/10.25267/Cuad investig fondos arch UCA.2021.i3.02 
En su expediente académico constan dos matrículas de honor en las asignaturas de Agricultura y Física, siete sobresalientes, seis notables y doce aprobados ${ }^{6}$. En ese periodo residía con sus padres en la Plaza del General Lachambre, actualmente conocida como Plaza del Teatro.

Figura 1. Certificación académica de los estudios de bachillerato de Fanny Medina presentada para su ingreso en la Facultad de Medicina de Cádiz en 1911

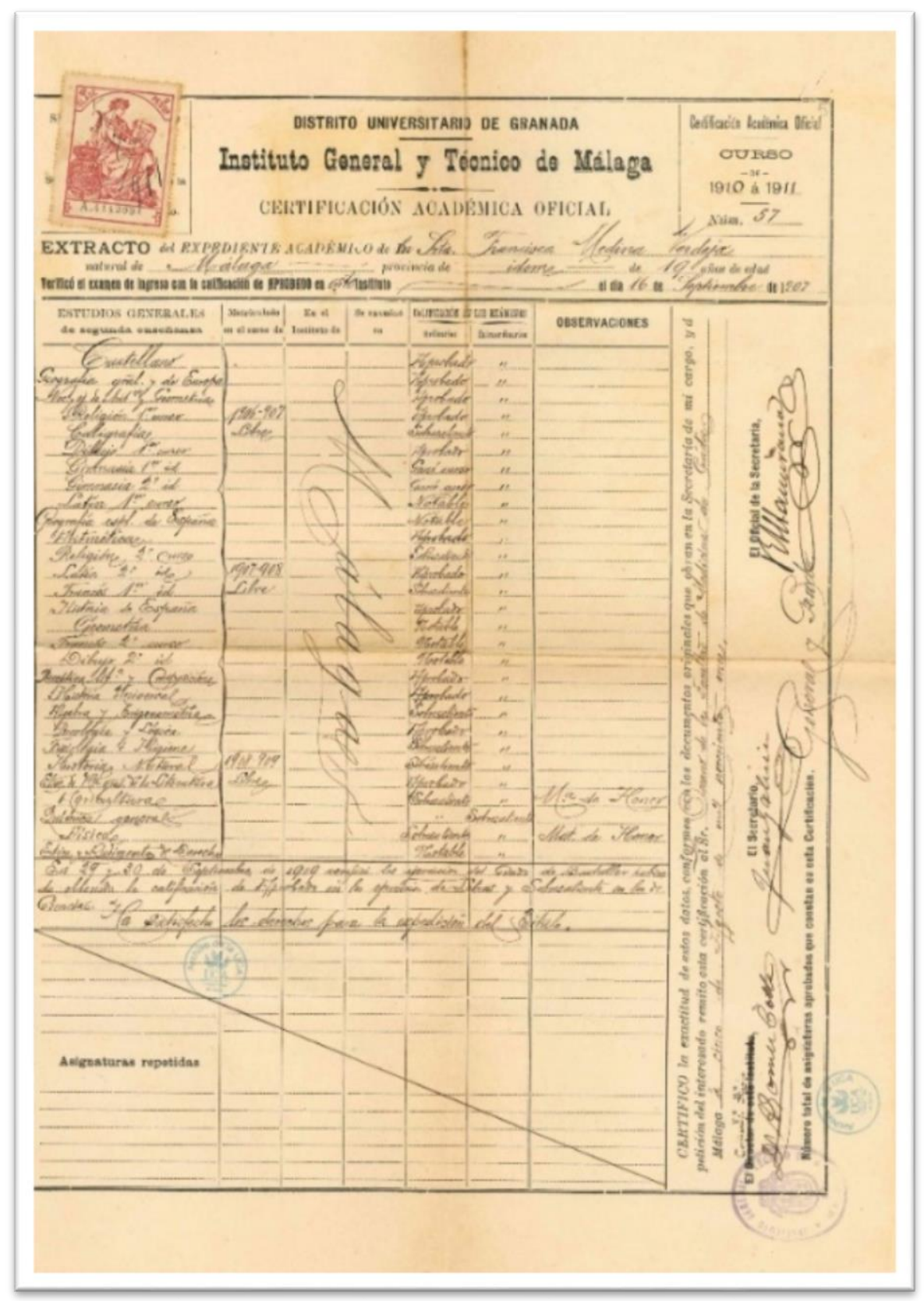

Fuente: Archivo de la Universidad de Cádiz (AUCA), C-236 (4) FM, folio 5

Concluido el Bachillerato retomó los estudios de Magisterio en el curso 1909-1910, aprobando varias asignaturas de los grados elemental y superior entre las convocatorias de

\footnotetext{
${ }^{6}$ Archivo del Instituto Vicente Espinel de Málaga (AIVEM), caja 44 y libros 64 y 327 . Hay un extracto de su expediente de bachillerato en la mencionada signatura del AGUMA. 
junio y septiembre. Suspendió Música y Canto, que aprobó tras repetir el examen, y Pedagogía. Es curioso que le costara superar la asignatura de Música, dado que para entonces había completado la carrera de Piano en el Conservatorio.

\section{FAnNy MEdina, ESTUdiante de MEdicina en CÁdIZ y dOCTORA EN MAdRID}

No conocemos la motivación de Fanny Medina para aspirar a ejercer como médica ni tampoco la causa de que eligiera Cádiz para matricularse frente a la otra opción más cercana, como era Granada, aunque sí es evidente que contaba con el apoyo de su familia. En septiembre de 1911, con veinte años recién cumplidos y el título de bachiller recién recibido, comenzó la carrera en la Facultad de Medicina de Cádiz. Hasta entonces en este centro habían cursado estudios superiores la citada Antonia Monreal y María del Mar Terrones Villanueva, aunque esta orientó su actividad posterior al periodismo.

Figura 2. Portada del expediente de Francisca Medina Verdeja en la Facultad de Medicina de Cádiz.

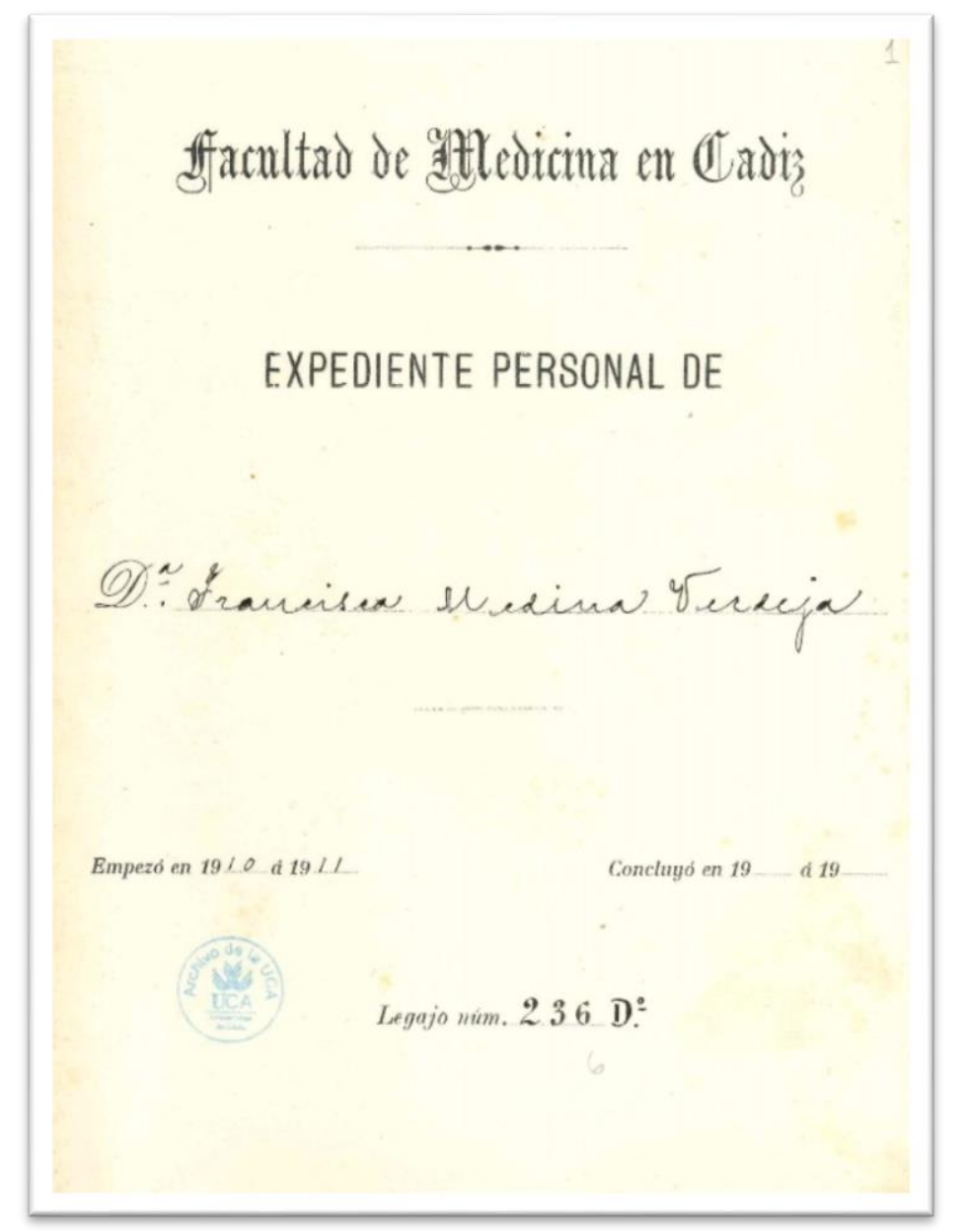

Fuente: Archivo de la Universidad de Cádiz (AUCA), C-236 (4) FM, portada, folio 1

En los meses anteriores Fanny se había estado preparando de forma privada para presentarse en la convocatoria de septiembre del curso 1910-1911, en la modalidad de enseñanza no oficial o libre, a las tres asignaturas que correspondían con el curso preparatorio 
de la Facultad de Ciencias: Física General, Mineralogía y Botánica y Zoología ${ }^{7}$. Aprobadas estas materias, en el curso siguiente, 1911-1912, solo se presentó a la asignatura de Química General, también del preparatorio de Ciencias, en la convocatoria no oficial del mes de junio, que superó con la calificación de notable. Aprovechó su estancia en Cádiz para matricularse en la Escuela Superior de Comercio con el único objetivo de dar validez académica a la enseñanza que había recibido privadamente de la lengua alemana con aplicación a la carrera de Medicina. Se examinó con calificación de sobresaliente ${ }^{8}$.

El diario El Correo de Cádiz se hizo eco de los resultados de estas pruebas en su número de 12 de junio de 1912:

Con notas inmejorables y llamando la atención de sus profesores se ha examinado de Química en esta Facultad de Medicina y de alemán en la de Artes e Industrias, la bella señorita malagueña Fany Medina, hija del rico propietario de aquella capital, D. Francisco. La Srta. de Medina que a su belleza une una gran inteligencia y amor al estudio, ha llamado mucho la atención por los exámenes verificados y en los que ha obtenido inmejorables notas. Según las noticias que tenemos, esta joven, acompañada de su familia, se establecerá en Cádiz para el próximo curso, pues desea matricularse como alumna oficial. Actualmente se encuentra en Chiclana donde pasará una temporada.

Con casi total seguridad esas primeras asignaturas del curso preparatorio las preparó en Málaga de forma particular y se desplazó a Cádiz exclusivamente para realizar los exámenes. Para el curso 1912-1913, tal como anunciaba el periódico, sí se matriculó como alumna oficial de la Facultad de Medicina para hacer las asignaturas de Anatomía, Histología e Histoquímica y Técnica Anatómica, que aprobó en mayo de 1913 con nota de sobresaliente en las tres.

En el curso 1913-1914 superó como alumna oficial las siguientes materias: segundo curso de Anatomía, segundo curso de Técnica Anatómica y Fisiología Humana, las dos primeras con sobresaliente en la convocatoria de mayo y la tercera en septiembre con un aprobado.

En agosto de 1914 el padre de Fanny elevó un escrito al Ayuntamiento de Málaga en el que solicitaba ayuda económica para poder continuar sus estudios ante la falta de recursos propios. Afirmaba que poseía el título de bachiller, que era maestra y profesora de música y que hablaba tres idiomas, además de haber aprobado ya los tres primeros cursos de la carrera en la facultad gaditana. Obtuvo del municipio la concesión de una pensión para que finalizara sus estudios de Medicina (Campos Luque 2001, p. 174). La solicitud fue presentada por su padre, Francisco Medina (El Popular, 20-8-1914).

La prensa malagueña trató la petición con gran atención debido a la excepcionalidad del caso. El Popular celebraba que fuera la primera hija de Málaga que emprendía la carrera de Medicina y animaba al Ayuntamiento a conceder la pensión solicitada a quien

salvando rancios prejuicios de sexo, sacrificando su esplendorosa juventud a una noble causa, posponiendo quizá el remanso tranquilo de un hogar sosegado y exclusivamente suyo, ahogando en su pecho amores que nacieron para morir en flor, por añadir un

\footnotetext{
${ }^{7}$ El expediente de Francisca Medina Verdeja en la Facultad de Medicina de Cádiz está en el Archivo de la Universidad de Cádiz (AUCA), C-236(4)-6 FM.

${ }^{8}$ AUCA. Expediente personal de Francisca Medina Verdeja, C-94-8 EC.

DOI del artículo: https://doi.org/10.25267/Cuad investig fondos arch UCA.2021.i3.02 
preciado galardón a su tierra, a su patria chica, por ser útil a la humanidad, por llevar el consuelo y la salud a otros seres, a otros semejantes suyos. Y por ser este caso quizá único en los anales de Málaga, si nosotros no estamos equivocados, por tratarse de una joven de talento, por significar un sacrificio elevado su decisión, debéis acceder a lo que de vosotros espera la bella Fanny [...]. Todos aplaudirán vuestra decisión y especialmente las bellas, que tanto saben agradecer los favores que se las hacen. La señorita Fanny es hermosa, digna de ser malagueña y victoriana; tiene talento, mucho talento y anhela ser útil a la humanidad (El Popular, 15-8-1914).

El autor de artículo, L. R. C. (seguramente el redactor Luis Rodríguez Cuevas), citaba precedentes de ayudas similares concedidas por otros ayuntamientos como Madrid, Barcelona, Valencia y Valladolid.

El semanario gráfico La Unión Ilustrada (20-9-1914) publicó una foto de Fanny con motivo de la concesión de la ayuda municipal, acompañada de este texto:

El Ayuntamiento de Málaga ha acordado entre generales aplausos, conceder una subvención a la señorita Fanny Medina, distinguidísima joven de excepcionales aptitudes que cursa con brillantez extraordinaria la carrera de Medicina en la Facultad de Cádiz. Fanny, que apenas cuenta 22 años, posee el título de bachiller en letras, habla tres idiomas y en la Facultad se distingue por su talento.

Figura 3. Fotografía de Fanny Medina como alumna de Medicina en Cádiz

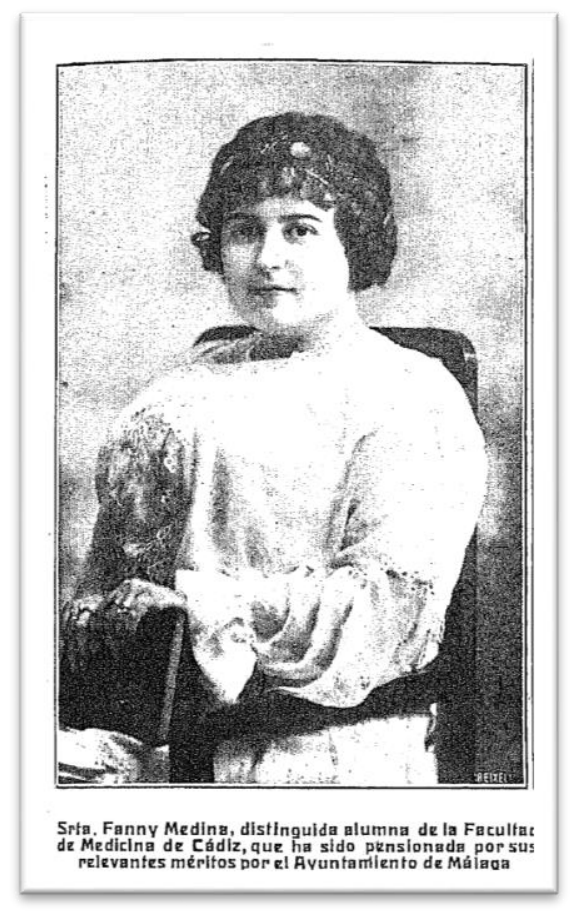

Fuente: La Unión Ilustrada (Málaga), 20 de septiembre de 1914

En febrero de 1915 Fanny dio una conferencia en el Centro Escolar Gaditano, círculo que había sido fundado por un grupo de estudiantes en 1911, abordando un tema social y político de máxima actualidad en aquel momento, puesto que solicitaba el indulto para los reos condenados a muerte por los sucesos de Benagalbón. En este pueblo cercano a Málaga se 
produjeron unos incidentes en marzo de 1914 por el temor de los vecinos a que se falsearan los resultados de las elecciones generales. El enfrentamiento se saldó con la muerte de un guardia civil y la detención de decenas de vecinos. Un matrimonio fue acusado de asesinato y condenado a la pena capital, hecho que dio lugar a una campaña de ámbito nacional impulsada por los republicanos para conseguir el indulto. En Cádiz, a raíz de la intervención de Fanny se formó una comisión para recoger adhesiones a la petición, que en esos meses estaba siendo apoyada en toda España por numerosas organizaciones, entidades y personalidades (Arcas Cubero 1985, pp. 477-478). La actualidad de la charla, y quizás el hecho de que fuera protagonizada por una mujer, hizo que fuera reseñada en medios de diferentes puntos del país, como el madrileño La Correspondencia de España (25-2-1915), el barcelonés La Vanguardia (25-2-1915) y el Diario de Córdoba (24-2-1915), siendo recogida con imágenes en La Unión Ilustrada (14-3-1915). Esta conferencia es el único testimonio que disponemos de la orientación política de Fanny, dando a entender sus simpatías por el republicanismo o, al menos, su cercanía a la causa de los condenados.

Figura 4. Fotografías de la conferencia de Fanny Medina en el Centro Escolar Gaditano

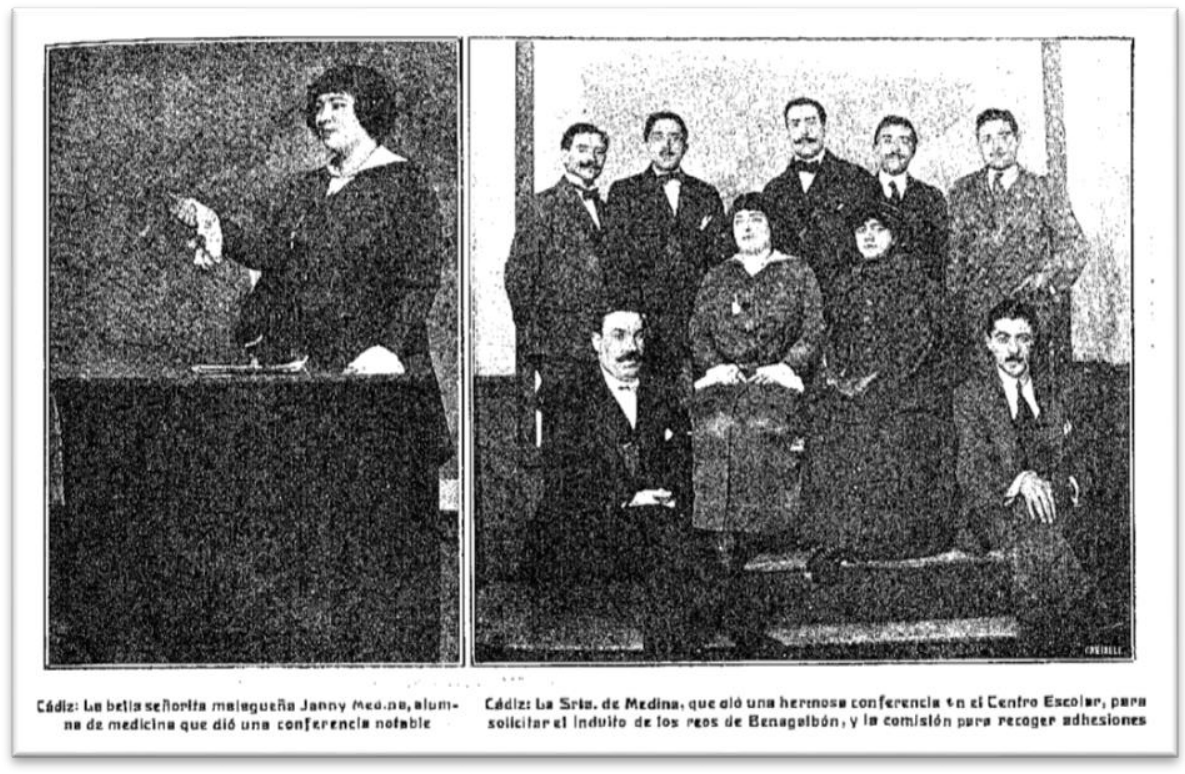

Fuente: La Unión Ilustrada (Málaga), 14 de marzo de 1915

Para el curso 1914-1915 se matriculó en Anatomía Patológica, Terapéutica y Patología General, dando como dirección en Cádiz la calle Virgili n. ${ }^{\circ}$ 7. En mayo de 1915 aprobó las tres materias con sobresaliente. Los recuerdos familiares transmitidos por la propia Fanny destacan que en estos años practicaba la natación, deporte que disfrutaba mucho, y en el que obtuvo varios premios. En cuanto a las clases en la Facultad gaditana, comentaba a sus descendientes que se sentaba en una mesa aparte del resto del alumnado, situada junto a la del catedrático.

El siguiente curso, 1915-1916, se inscribió en cinco asignaturas: Patología Quirúrgica (primer curso), Patología Médica (primer curso), Obstetricia, Anatomía Topográfica y Oftalmología. Suspendió en mayo Anatomía Topográfica, que tuvo que dejar para septiembre 
junto a Patología Médica. En Obstetricia y Oftalmología obtuvo sobresaliente. En mayo de 1916 solicitó la realización de las prácticas clínicas como alumna interna con destino a Obstetricia.

Fanny contó a sus descendientes que había suspendido una asignatura en la carrera (la de Anatomía Topográfica, como hemos visto) porque el profesor la pretendió y ella rechazó su propuesta. Después de su protesta, fue examinada ante un tribunal. Lo cierto es que en el acta del primer examen, celebrado el 29 de mayo, solo aparece la firma del profesor, mientras que el segundo, que tuvo lugar el 24 de septiembre, fue realizado ante un tribunal formado por tres miembros ${ }^{9}$.

Figura 5. Calificaciones de Fanny Medina en el curso 1915-1916

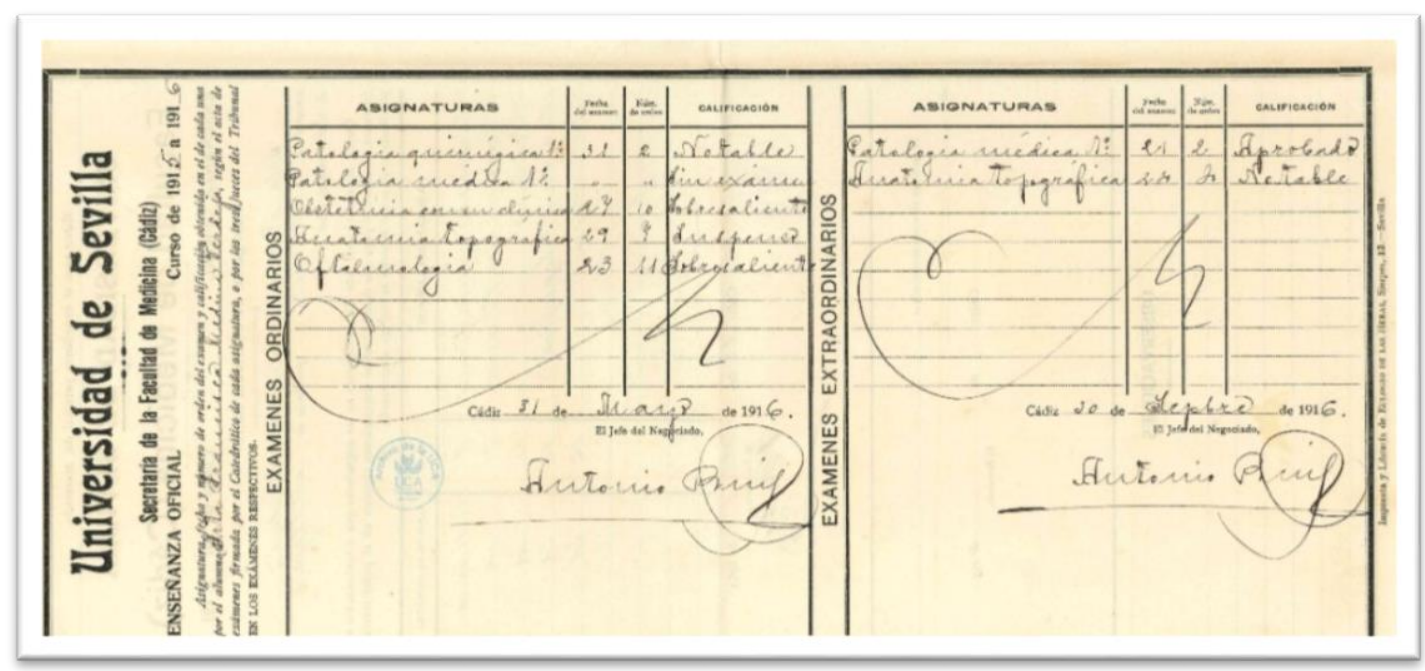

Fuente: Archivo de la Universidad de Cádiz (AUCA). C-236 (4) FM

En el curso 1916-1917 continuó como alumna oficial, facilitando como dirección la calle Sagasta n. ${ }^{\circ}$ 28. Obtuvo sobresaliente en Ginecología, notable en Otorrinolaringología y Patología Quirúrgica (segundo curso) y aprobado en Patología Médica (segundo curso) y Enfermedades de la Infancia.

Finalmente, en el curso 1917-1918 superó las últimas asignaturas que le faltaban para concluir la carrera: Patología Quirúrgica (tercer curso) con sobresaliente y aprobados en Patología Médica (tercer curso), Higiene, Medicina Legal y Dermatología y Sifiliografía. Después de los exámenes de mayo, alcanzó la licenciatura en la Facultad de Cádiz el 22 de junio de 1918 con la calificación de sobresaliente ${ }^{10}$. Su foto con birrete apareció en La Unión

\footnotetext{
${ }^{9}$ AUCA. Libro de actas de exámenes oficiales y no oficiales de septiembre de la Facultad de Medicina en Cádiz, L-51 FM; Libro de actas de exámenes oficiales y no oficiales de enero, mayo y junio de la Facultad de Medicina en Cádiz, L-52 FM.

Agradezco a Clemencia de la Cruz y a $\mathbf{M}^{\mathrm{a}}$. Rosario Marín la búsqueda realizada en los libros de actas de sesiones del Claustro y en los registros de correspondencia de la Facultad de Medicina, sin que conste en los mismos ninguna queja o reclamación de Francisca Medina.

${ }^{10}$ El título fue registrado en el Colegio de Barcelona en 1925. Arxiu Històric del Col·legi de Metges de Barcelona (AHCoMB), Registro de títulos de la Subdelegación Médica de las Afueras de Barcelona.
} 
Ilustrada el 26 de septiembre de 1918, junto con el anuncio de que se trasladaba a Madrid para realizar el curso de doctorado. Los alumnos del último año de Medicina de aquel curso organizaron una cena de homenaje en la terraza de la Cervecería Inglesa a Celestino Párraga, decano de la Facultad gaditana. Fanny no asistió, pero mostró su adhesión al homenaje con la remisión de una carta «redactada en términos afectuosísimos» (El Correo de Cádiz, 7-4-1918).

Figura 6. Fotografía de Fanny Medina una vez obtenida la licenciatura en Medicina

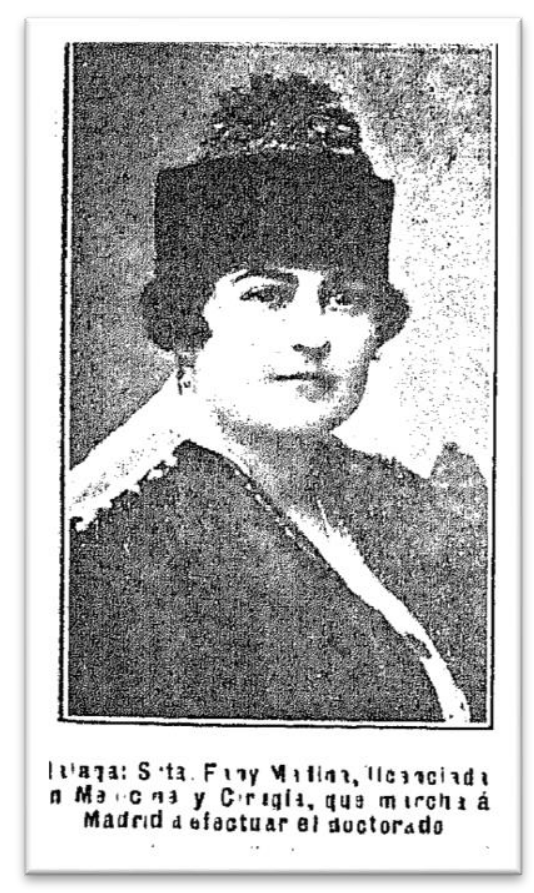

Fuente: La Unión Ilustrada (Málaga), 26 de septiembre de 1918

Fanny decidió aprovechar para finalizar al mismo tiempo la carrera de Magisterio que había dejado incompleta antes de iniciar la de Medicina. Le quedaban dos asignaturas, Pedagogía y Economía Doméstica, para obtener el título de Maestra de Primera Enseñanza con arreglo al real decreto de 30 de agosto de 1914 que había reorganizado estos estudios y establecido un nuevo plan académico. No tuvo que practicar los ejercicios de reválida al acogerse a los beneficios del real decreto de 10 de marzo de 1917, que establecía que era suficiente con la aprobación de las asignaturas comprendidas en el plan de estudios, y en junio de 1918 se gestionó el expediente para la concesión del título por la Escuela Normal de Maestras de Cádiz ${ }^{11}$.

Mientras Fanny concluía de forma simultánea las carreras de Medicina y Magisterio, su padre ya estaba gestionando su traslado a Madrid. En el mes de abril Francisco Medina visitó la Residencia de Señoritas y se entrevistó con María de Maeztu para informarse de las condiciones para que su hija se alojara en la misma durante el curso de doctorado. En una carta que envió a ésta, fechada en Málaga el 11 de mayo, afirmaba que no había presentado la solicitud en aquel momento «porque como mi hija no se ha separado durante sus estudios del lado de su madre, no tenía seguridad de poderla convencer de que estando en esa residencia

${ }^{11}$ AUCA. Expediente personal de Francisca Medina Verdeja, C-376-13 EN.

DOI del artículo:

https://doi.org/10.25267/Cuad investig fondos arch UCA.2021.i3.02 
no eran precisos los cuidados de su dicha madre». Una vez recibido el impreso el 28 de ese mes Francisco Medina remitió la documentación necesaria con un pago mensual de 110,50 pesetas en concepto de gastos de alojamiento, asistencia médica y estudios ${ }^{12}$.

Por tanto, en el curso siguiente Fanny marchó a Madrid para proseguir con el doctorado, alojándose en la Residencia de Señoritas que dirigía María de Maeztu desde su creación en 1915 y que estaba en la calle Fortuny, dentro del complejo de la Residencia de Estudiantes. Fue una de las ocho andaluzas residentes que cursaron estudios de doctorado y una de las tres que lo hicieron en la Facultad de Medicina, junto a Cecilia García de la Cosa y Nieves González del Barrio (Lemus López 2017, p. 32). Desconocemos cuánto tiempo permaneció en la Residencia, pero según los testimonios de sus familiares fue una estancia breve, ya que no se adaptó a las normas de la institución y pronto tuvo diferencias con la misma María de Maeztu. La directora ejercía un firme control cotidiano de las chicas alojadas, de acuerdo con las normas sociales de la época (López Cobo 2010, p. 534). Como resultado, Fanny decidió trasladarse a una pensión situada en la calle del Prado, en la que conoció a su futuro marido ${ }^{13}$.

Tardó dos años en completar su tesis doctoral, que defendió en junio de 1920. Se graduó como doctora en la Universidad Central con la calificación de sobresaliente por unanimidad presentando la memoria titulada Importancia ginecológica y aplicación terapéutica del radio en los procesos neoplásicos, que realizó bajo la dirección del doctor Sebastián Recasens, prestigioso catedrático y decano de la Facultad de Medicina de dicha Universidad. Recasens era autor de los principales manuales de Obstetricia y Ginecología que se utilizaban entonces en las facultades españolas y fue el introductor en el país de las primeras técnicas que empleaban radioterapia para combatir el cáncer (Matilla 1987, p. 123), cuestión abordada precisamente por Fanny Medina en su tesis. La noticia de la nueva doctora en Medicina fue recogida por varios medios nacionales, como La Libertad (24-6-1920), El Liberal (25-6-1920) y La Correspondencia de España (26-6-1920), en todos los casos con el mismo texto.

\section{COLEGIACIÓN Y EJERCICIO DE LA PROFESIÓN}

En los primeros días de enero de 1921 Fanny contrajo matrimonio con José María Bermejillo y Pelayo en la iglesia de la Victoria de Málaga, ante el altar de la patrona de la ciudad. Actuaron de padrinos don José García Guerrero y la señorita Celia Bermejillo, hermana del novio. Firmaron el acta matrimonial como testigos Salvador González Anaya, Antonio de las Peñas, Manuel Verdejo y Juan Miró Multró. Los recién casados salieron de viaje hacia Córdoba, para continuar seguidamente hacia Madrid y Bilbao (La Correspondencia de España, 7-1-1921). Los recuerdos familiares hablan de un viaje al Marruecos francés, durante el cual Fanny tuvo que improvisar una consulta ginecológica en un hotel para solventar la pérdida de dinero sufrida por un robo. La foto del enlace apareció publicada en varios medios gráficos, como La Unión Ilustrada (26-1-1921) y el madrileño Nuevo Mundo (4-21921).

\footnotetext{
12 Archivo de la Residencia de Señoritas, Fundación José Ortega y Gasset-Gregorio Marañón (ARS), signaturas $38 / 27 / 21$ y $38 / 27 / 22$.

${ }^{13}$ Información facilitada por su nieto, Manuel Pérez de Petinto, en conversación mantenida el 26-9-2020.

DOI del artículo:

https://doi.org/10.25267/Cuad investig fondos arch UCA.2021.i3.02 
El novio ostentaba desde 1916 el cargo honorífico de montero de guarda y cámara de Sus Majestades los Reyes de España, cuyo uniforme vistió en la ceremonia nupcial. El padre del marido de Fanny, Félix de Bermejillo, había sido alcalde de Espinosa de los Monteros (Burgos), localidad natal de José María, quien había cursado estudios en la Facultad de Ciencias de la Universidad Central entre 1904 y $1908^{14}$.

Medina fue la primera mujer en inscribirse en el Colegio de Médicos malagueño, aunque su expediente no se conserva y por este motivo no es posible precisar la fecha de su colegiación (Prados Carmona 2006, p. 354). Por los anuncios que publicaba en la prensa local sabemos que tenía consulta ginecológica abierta en un piso de la calle Comedias al menos desde septiembre de 1921 (el primer anuncio detectado apareció en La Unión Mercantil el 2 de septiembre de ese año). Estos anuncios siguieron apareciendo en los meses siguientes. La apertura del «consultorio para señoras» de la doctora Fanny Medina tuvo que ser, en todo caso, posterior a su inscripción en el Colegio. Se daba la circunstancia de que Fanny estaba embarazada entonces, puesto que su primera hija, María Celia, nació el 31 de enero de 1922.

Figura 7. Anuncio del consultorio ginecológico de Fanny Medina publicado en 1921

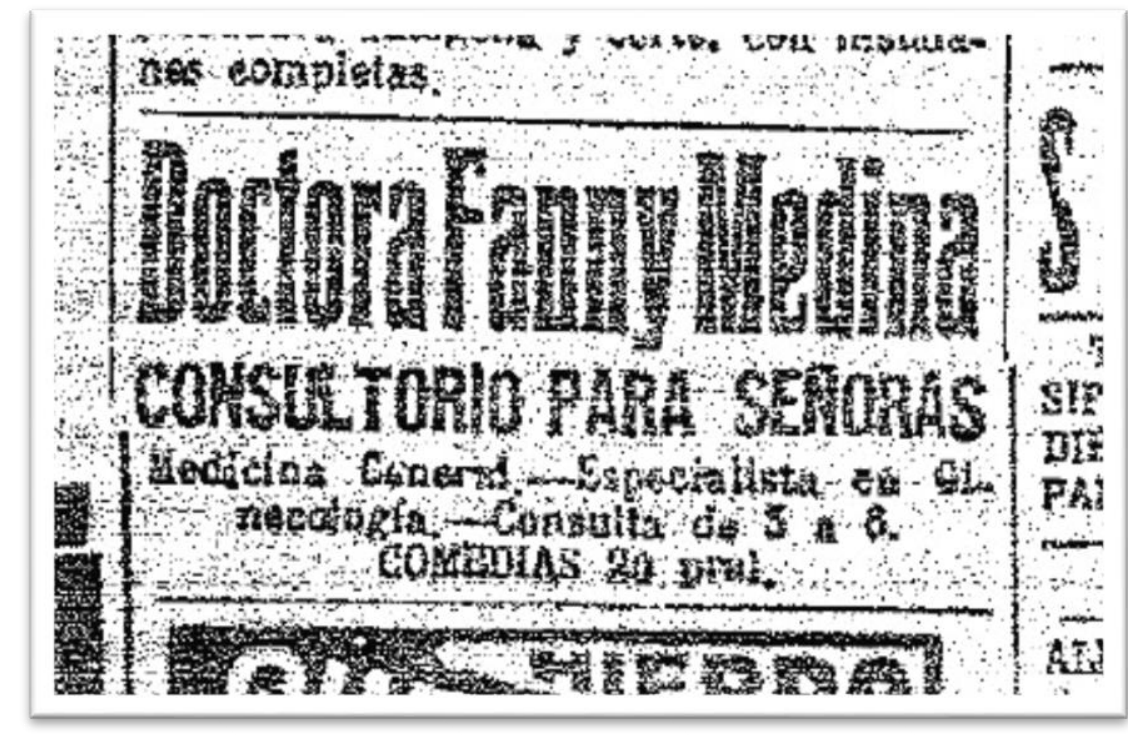

Fuente: La Unión Mercantil (Málaga), 2 de septiembre de 1921

En 1925 se dio de baja en el Colegio de Málaga por traslado a Barcelona (Prados Carmona 2006, pp. 137 y 354), aunque su estancia en la capital catalana fue breve. En el registro de títulos del Colegio de Médicos de Barcelona consta que se dio de alta el 24 de octubre de 1925 después de ser admitida en la sesión celebrada cuatro días antes ${ }^{15}$. Ejerció en la ciudad condal durante un par de años, ya que en octubre de 1927 se le dio de baja por «dejar de ejercer». En la documentación colegial constan dos direcciones de Fanny en la ciudad condal: Aribau n. ${ }^{\circ}$

\footnotetext{
${ }^{14}$ Archivo Histórico Nacional (AHN), signaturas FC - Mo JUSTICIA - MONTEROS CÁMARA, caja 614, exp. 80 y UNIVERSIDADES, caja 5.329, exp. 20. En: http://pares.mcu.es/Pares/bermejillo+pelayo

15 Arxiu Històric del Col-legi de Metges de Barcelona (AHCoMB), Registro de títulos de la Subdelegación Médica de las Afueras de Barcelona. Estos datos me han sido proporcionados por la archivera Sara Fajula Colom, a quien agradezco su amabilidad e interés.
}

DOI del artículo:

https://doi.org/10.25267/Cuad investig fondos arch UCA.2021.i3.02 
127, en el Ensanche (cambio de domicilio informado en mayo de 1926) y Avenida República Argentina n. ${ }^{\circ} 15$ (cuando se le da de baja en octubre de 1927) ${ }^{16}$.

El regreso a su ciudad natal coincidió con el nacimiento de Francisco, su segundo hijo. Retornó al ejercicio profesional en 1928 (Ortiz Gómez 1985-86, p. 362) y en el Anuario General de Málaga de 1930, editado por Valero Enfedaque, figura con consulta abierta en la calle Tejón y Rodríguez n. ${ }^{\circ}$ 31, aunque en el listado de médicos aparece como «Francisco». Este mismo error se repite en la Guía Popular de Málaga editada en 1933, en la que se incluye entre los médicos a «don Francisco Medina Verdeja».

El padrón municipal de 1930 nos confirma la presencia de Fanny y sus dos hijos residiendo con su padre, Francisco Medina, en la calle Montaño (entonces denominada Arturo Reyes) n. ${ }^{\circ}$ 4-8. Fanny figura como doctora en Medicina ${ }^{17} \mathrm{y}$, aunque consta como casada, sabemos por los testimonios de sus nietos que ya estaba separada de su marido.

En los primeros años treinta, Fanny Medina vuelve a anunciar en la prensa su «consultorio para señoras», como «especialista en enfermedades de la mujer y niños» (El Popular, 19-31932), manteniendo la dirección de Tejón y Rodríguez n. ${ }^{\circ}$ 31. Según datos del Anuario Médico de España de 1930-1931, citados por Teresa Ortiz, entonces había colegiadas en España un total de 51 médicas ejercientes. En 1928 se había fundado la Asociación de Médicas Españolas por un grupo de quince licenciadas y doctoras. Fue promovida por Elena Soriano, también discípula del doctor Recasens y que se había doctorado el mismo año que Fanny, en 1920, aunque la malagueña no llegó a inscribirse en dicha asociación (Ortiz Gómez 1988). Soriano, que ejerció como oftalmóloga, ya había sido la primera presidenta de la Juventud Universitaria Femenina, constituida también en 1920.

En agosto de 1932, pocos días después de cumplir 41 años, Fanny tuvo que afrontar el que tuvo que ser uno de los peores momentos de su vida: el suicidio de su padre. Era muy conocido en la ciudad por su labor comercial y por haber regentado un establecimiento de bebidas denominado «Los Gabrieles» (Diario de Málaga, 30-8-1932). El cadáver de Francisco Medina fue localizado flotando en las aguas del puerto (El Cronista, 29-8-1932). Fanny acudió a identificarlo al depósito judicial, ante la sospecha de que podía tratarse de su padre, que se había marchado de su casa tres días antes dejando una carta anunciando su propósito suicida (El Popular, 30-8-1932). La junta directiva del Colegio de Médicos transmitió a la doctora Medina el pésame por la muerte de su padre en su sesión del 1 de septiembre (Prados Carmona 2006, p. 354).

La siguiente información de la que disponemos sobre la actividad profesional de Fanny procede de la sesión de la Junta General del Colegio de Médicos celebrada el 26 de enero de 1935, en la que fue denunciada por otro médico, Antonio Alcalá López, por ejercer la profesión sin pagar las cuotas colegiales ni la contribución (Prados Carmona 2006, p. 442).

A partir de ese año dejamos de encontrar información sobre Fanny Medina y todo lo que sabemos de su trayectoria posterior procede de los testimonios de sus nietos Manuel y Francisco Javier Pérez de Petinto. Al parecer, hacia 1935 Fanny decidió trasladarse a Madrid

\footnotetext{
${ }^{16}$ Butlletí del Sindicat de Metges de Catalunya, n. ${ }^{\circ}$ 64, 1925, p. 40. Boletín del Colegio Oficial de Médicos de la Provincia de Barcelona, n. ${ }^{\circ} 3,1926$, p. 12, y n. $^{\circ} 17,1927$, pp. 19 y 28.

${ }^{17}$ Archivo Municipal de Málaga (AMM), Padrón municipal de 1930, libro 1.484, f. 88v.

DOI del artículo:

https://doi.org/10.25267/Cuad investig fondos arch UCA.2021.i3.02 
con sus hijos, aún menores de edad. En la capital pasó la Guerra Civil y contó con el apoyo del doctor Manuel Bermejillo, primo de su marido. Tras su paso por la política (fue diputado en 1936 por Burgos en la candidatura de la CEDA), Bermejillo fue catedrático de Patología de la Universidad Complutense y presidente de la Real Academia de Medicina, entre otros muchos cargos.

Fanny Medina continuó ejerciendo la Medicina en las consultas que tuvo en sus domicilios de las calles Belén y Mauricio Legendre, según las informaciones proporcionadas por sus familiares. Falleció en Madrid el 23 de mayo de 1977, a la edad de 85 años.

\section{CONCLUSIÓN}

Pilar Ballarín (1994) escribía hace años que la historia de la educación de las mujeres se había movido en un nivel primario, en el que existía una auténtica «necesidad de nombrar, de convertir a las mujeres anónimas en sujetos singulares protagonistas de la vida pública». Un cuarto de siglo después vemos que todavía sigue siendo necesaria esa labor de rescate y recuperación de personalidades desconocidas que en su momento fueron pioneras. Como han indicado algunas autoras, la biografía sigue siendo un método de análisis valioso a la hora de estudiar la historia de las Ciencias desde una perspectiva de género (Santesmases, Cabré y Ortiz 2017).

En este artículo se presenta una primera aproximación a la biografía de una de las primeras mujeres que estudió Medicina en Andalucía y, posiblemente, la primera que le dio utilidad a su titulación para ejercer la profesión de médica. Al menos, los datos conocidos y documentados así permiten afirmarlo, ya que la primera noticia de su trabajo data de 1921, dos años antes que la colegiación de Eudoxia Píriz en Granada. Francisca Medina Verdeja prolongó esta dedicación durante toda su vida, según los testimonios familiares, al contrario que Píriz, que dejó de ejercer después de la Guerra Civil. No conocemos ninguna publicación de Medina y todavía quedan muchos aspectos que investigar acerca de ella y de sus compañeras pioneras, que forman los primeros nombres de una larga lista de profesionales que, en nuestros días, se enfrentan a uno de los mayores retos de la Medicina en el último siglo.

Quiero agradecer las facilidades y la amabilidad con que han sido atendidas mis peticiones de información por parte del personal de los siguientes centros documentales: Archivo de la Universidad de Cádiz, Archivo General de la Universidad de Málaga, Archivo del Colegio de Médicos de Barcelona y Fundación Ortega-Marañón de Madrid.

\section{REFERENCIAS BIBLIOGRÁFICAS}

ArCAs Cubero, F., 1985. El republicanismo malagueño durante la Restauración (18751923). Córdoba: Ayuntamiento de Córdoba.

BALlarín Domingo, P., 1994. La educación contemporánea de las mujeres. En: GUEREÑA, J. L., TIANA FERrer, A. Y RUIZ BERrio, J., coords. Historia de la educación en la España contemporánea: diez años de investigación. Madrid: Ministerio de Educación, Cultura y Deporte, pp. 173-190. 
Barranco Castillo, E., [en línea]. Eudoxia Píriz Diego. Diccionario Biográfico de la Real Academia de la Historia [Consulta: 10 septiembre 2020]. Disponible en http://dbe.rah.es/biografias/90437/eudoxia-piriz-diego

CABRÉ I PAIRET, M., 2001. Autoridad e historia: El proyecto historiográfico de las médicas estadounidenses, 1925-1940. [En línea]. En: Asparkia. Investigació feminista, n. ${ }^{\circ} 12$, pp. 113-122. [Consulta: 23 julio 2020]. Disponible en: http://www.erevistes.uji.es/index.php/asparkia/article/view/888

Campos LuQue, C., 2001. Mercado de trabajo y género en Málaga durante la crisis de la Restauración. Granada: Universidad de Granada.

FleChA GARCÍA, C., 1996. Las primeras universitarias en España, 1872-1910. Madrid: Narcea.

Flecha García, C., 1998. Educación y trabajo de las mujeres en la Restauración. En: SALCEDO, M. J., comp. Estrategias laborales femeninas: Trabajo, hogares y educación. Málaga: Diputación Provincial de Málaga, pp. 179-197.

GARCía BARRAnCO, M. y BARRAnCo CASTILlo, E., 2002. Eudoxia Píriz Diego (1893-1980), recuperando una mujer para la historia. En: Las Mujeres en la Historia de Andalucia. Córdoba: Cajasur, tomo II, pp. 73-78. Texto disponible en: http://www.historiamujeres.es/vidas/EUDOXIA_PIRIZ.pdf

GuIL BozAL, A. Y FleChA GARCíA, C., 2015. Universitarias en España: De los inicios a la actualidad. [En línea]. En: Revista Historia de la Educación Latinoamericana, n. ${ }^{\circ}$ 17, pp. 125-148. [Consulta: 22 agosto 2020]. Disponible en: https://doi.org/10.19053/01227238.3303

LEMUS LÓPEZ, E., 2017. Llegar a la Universidad y a la gran ciudad 'en femenino'. Las estudiantes andaluzas en la Residencia de Señoritas. [En línea]. Sevilla: Centro de Estudios Andaluces. [Consulta: 17 junio 2020]. Disponible en: https://centrodeestudiosandaluces.es/publicaciones/llegar-a-la-universidad-y-a-lagran-ciudad-en-femenino-las-estudiantes-andaluzas-en-la-residencia-de-senoritas

LÓPEZ CовO, A., 2010. El «espíritu de la casa» en la Residencia de Estudiantes. Características específicas del grupo de señoritas. En: SÁnchez Ron, J. M. Y GARCía-VElasco, J., coords. 100 años de la JAE. La Junta para Ampliación de Estudios e Investigaciones Científicas en su centenario. Madrid: Residencia de Estudiantes, tomo II, pp. 530-549.

LÓPEZ MARINAS, J. M., 2015-2016. Málaga y la malagueña en la música clásica. En: Isla de Arriarán, n. ${ }^{\circ} 42-43$, pp. 229-270.

Magallón PoRTAlÉs, C., 2011. Su libertad y su esfuerzo nos abrieron espacios: pioneras españolas en las ciencias experimentales en el primer tercio del siglo XX. [En línea]. En: Tabanque: Revista pedagógica, $\mathrm{n}^{\circ}$ 24, pp. 175-189. [Consulta: 2 septiembre 2020]. Disponible en: http://hdl.handle.net/11162/182683

Matilla, V., 1987. 202 biografías académicas. Madrid: Real Academia Nacional de Medicina.

OrTIZ GómEz, T., 1985-1986. La mujer como profesional de la Medicina en la España contemporánea: El caso de Andalucía, 1898-1981. En: Dynamis, vol. 5-6, pp. 343-366.

OrTiz GómEZ, T., 1988. La Asociación de Médicas Españolas (1928-1936) y su fundadora, doctora Elisa Soriano (1891-1964). En. AA.VV., Actas del VIII Congreso Nacional de Historia de la Medicina. Murcia, pp. 595-606.

Prados Carmona, G., 2006. Málaga y sus médicos. De los "felices 20” a 1936. Málaga: Grupo Editorial 33.

RODRíGUEZ LóPEZ, C., 2010. Experiencias universitarias en torno a 1910. En el centenario del acceso de la mujer a los estudios universitarios. [En línea]. En: Participación

DOI del artículo:

https://doi.org/10.25267/Cuad investig fondos arch UCA.2021.i3.02 
Educativa, n. ${ }^{\circ}$ 15, pp. 209-219. [Consulta: 11 octubre 2020]. Disponible en: https://sede.educacion.gob.es/publiventa/detalle.action?cod=14205

SANTESMASES, M. J.; CABRÉ I PAiRet, M. y ORTIZ Gómez, T., 2017. Feminismos biográficos: aportaciones desde la Historia de la Ciencia. [En línea]. En: Arenal, n. ${ }^{\circ} 24: 2$, pp. 379 404. [Consulta: 14 septiembre 2020]. Disponible en: https://revistaseug.ugr.es/index.php/arenal/article/view/6303

VÁZQUEZ RAMIL, R., 2012. Mujeres y educación en la España Contemporánea. La Institución Libre de Enseñanza y la Residencia de Señoritas de Madrid. Madrid: Akal. 